\title{
Paraoxonase-1 and other factors related to oxidative stress in psoriasis
}

\author{
Magdalena Oszukowska, Magdalena Kozłowska, Andrzej Kaszuba \\ Department of Dermatology, Pediatric and Oncological Dermatology, Medical University of Lodz, Lodz, Poland \\ Adv Dermatol Allergol 2020; XXXVII (1): 92-96 \\ DOI: https://doi.org/10.5114/ada.2020.93386
}

\begin{abstract}
Introduction: Psoriasis is considered as a risk factor for atherosclerosis and contributes to myocardial infarction, ischemic heart disease and brain stroke.

Aim: To estimate the atherogenic potential of psoriasis by analysing antioxidative and prooxidative factors (paraoxonase-1, $\alpha$-tocopherol, uric acid, homocysteine), compare levels of these parameters between groups of psoriatic patients and healthy individuals as well as to analyse the impact of psoriasis severity and duration on the factors under the study and to define correlation between the marked factors and patients' lifestyles, body mass index (BMI) and abdominal circumference.

Material and methods: The investigated group consisted of 66 patients with psoriasis vulgaris, while the control group comprised 30 persons. Both groups were comparable as regards their age, sex and BMI as well as abdominal circumference.

Results: A significantly lower activity of paraoxonase-1 $(p<0.001)$, level of tocopherol $(p<0.05)$ and significantly higher concentration of homocysteine $(p<0.01)$, uric acid $(p<0.05)$ were found in patients with psoriasis as compared to the reference group. A higher homocysteine level occurs in patients with a negative family history of psoriasis $(p<0.05)$. In the group of patients with psoriasis and metabolic syndrome, the uric acid level was significantly higher $(p<0.05)$. Concentration of uric acid correlated negatively with the abdominal circumference value $(p<0.001)$. Conclusions: Psoriasis promotes arteriosclerosis development by decreasing the levels of antiatherogenic and increasing the levels of proatherogenic agents. Adverse changes in psoriatic patients involve activity of paraoxonase-1, levels of $\alpha$-tocopherol, uric acid, homocysteine as compared to healthy individuals selected by their age, BMI and abdominal circumference value.
\end{abstract}

Key words: psoriasis, paraoxonase-1, $\alpha$-tocopherol, homocysteine, uric acid.

\section{Introduction}

Oxidative stress is related to a disturbance in the balance between antioxidants and prooxidants when the system removing oxygen free radicals cannot keep up with their production. It is a physiological process related to an inflammation and contributes to the development of macromolecular damages. An atom or a particle which has an unpaired valence electron is called a free radical. A low reactive oxygen species (ROS) level is necessary for the proper functioning of intercellular signalling and cellular function, whereas its high level is a pathological condition that results in modification of nucleic acids, protein or lipids, which in turn may lead to cell death. Mechanisms preventing oxidation include small molecules circulating in the serum (e.g. vitamin E, C, urates, bilirubin, creatinine) and enzymes catabolizing oxidized lipids (e.g. paraoxonase) [1-3].

\section{Aim}

The aim of the study was to assess the atherogenic potential of psoriasis by analysing antioxidative and prooxidative factors (paraoxonase- $1, \alpha$-tocopherol, uric acid, homocysteine), compare levels of these parameters between groups of psoriatic patients and healthy individuals of similar age, Body mass index (BMI) and abdominal circumference, as well as to analyse the impact of psoriasis severity and duration on the factors under the study and to define correlation between the marked

Address for correspondence: Prof. Andrzej Kaszuba MD, PhD, Department of Dermatology, Pediatric and Oncological Dermatology, Medical University of Lodz, 1/5 Kniaziewicza St, 91-347 Lodz, Poland, phone: +48 42651 10 72, e-mail: andrzej.kaszuba@umed.lodz.pl Received: 13.07.2018, accepted: 1.09.2018. 
factors and patients' lifestyles, their BMI and abdominal circumference.

\section{Material and methods}

The case-control study was conducted in 96 persons, including 47 females and 49 males. The sample group included 66 patients (31 females and 35 males) with clinically diagnosed vulgar psoriasis (psoriasis vulgaris), aged between 21 and 73 years, hospitalized in 2014-2015 in the Dermatology Department in Lodz. The reference group included 30 clinically healthy individuals (16 females and 14 males) aged between 30 and 70 years. The reference group was smaller than the sample group because the healthy individuals were to compare with a part of the patients with psoriasis depending of the severity of the pathological changes (according to Psoriasis Area and Severity Index (PASI) score):

- Psoriatic patients with PASI score equal to or lower than 10 points (21 patients - 31.8\%).

- Psoriatic patients with PASI score higher than 10 points (45 patients $-68.2 \%$ ).

Both groups were comparable as regards their age, sex and BMI as well as abdominal circumference. The patients participating in the study were diagnosed with vulgar psoriasis based on clinical examination.

The study excluded patients with:

1) symptoms of infections present on admission to the Clinic (fever, sore throat, cough, dysuria symptoms),

2) neoplastic diseases,

3) chronic diseases of the liver, pancreas, infectious diseases, heart failure etc.,

4) abnormal results of TSH, fT3 or fT4 tests,

5) megaloblastic anaemia

and patients:

6) taking medications affecting lipid metabolism, concentration of the uric acid or homocysteine, including statin, treated with cyclosporine A, methotrexate, sulfasalazine and retinoids,

7) abusing alcohol (drinking more than 10-12 g of ethanol a day which contributes to the development of alcoholic liver disease),

8) under the age of 18 years.

In the past some of the patients were treated with methotrexate, cyclosporine $A$, retinoids and received PUVA therapy, however they ceased to apply these treatment methods at least 3 months preceding the trial.

The trial was conducted under an approval given by the Bioethical Committee of the Medical University of Lodz. All participants of the trial were aware of its subject matter and gave their voluntary consent to participate in it.

A detailed history on the course of psoriasis was taken from each patient which included the following information: age of the patient, duration of the disease, frequency of relapses, location of skin lesions, family history, and pruritus exacerbation. Moreover, the questionnaire included information on coexisting diseases, medications taken, tobacco use, alcohol drinking habits and a diet applied.

A detailed medical history related to previous diseases and illnesses and hereditary factors was also taken from healthy individuals included in the reference group.

All trial participants underwent a detailed physical examination (including assessment of their body mass, height, BMI and abdominal circumference) and dermatological examination.

Venous blood samples were taken from fasting patients qualified for the trial between 7 and 8 a.m., in the amount of $8 \mathrm{ml}$ to a tube for blood clot and $4 \mathrm{ml}$ to a tube with EDTA at one time.

\section{Statistical analysis}

Statistical analyses were performed using program Statistica 10 (JPZP601E504326AR-9) with a significance level set at $p<0.05$. Student's t-test, Mann-Whitney $U$ test and Spearman's rank correlation coefficient were applied. The results were expressed as the mean \pm standard deviation.

\section{Results}

Based on a statistical analysis of the results it was established that in psoriatic patients there occurs a lower activity of paraoxonase-1 (PON-1) $(p<0.001)$ and a lower level of tocopherol $(p<0.05)$ as compared to the reference group (Table 1). No differences in the activity or level of these parameters dependent on PASI score, metabolic syndrome presence or lifestyle were observed. Individuals with a positive family history of psoriasis had a higher PON-1 activity as compared to patients with a negative family history of the disease. Moreover, the correlation between PON-1 activity and the duration of psoriasis was positive $(p<0.05)$.

The statistical analysis on the homocysteine level shows that its level was significantly higher in psoriatic patients, both in males and females, as compared to healthy individuals $(p<0.001)$. The homocysteine level was not affected by metabolic syndrome diagnosis, PASI score, diet or tobacco smoking. A higher homocysteine level occurs, however, in patients with a negative family history of psoriasis as compared to those patients who had psoriasis cases in their families $(p<0.05)$.

The uric acid level in the group of psoriatic patients was significantly higher than in the control group $(p<0.05)$. When analysing the uric acid level in the group of females and males separately, it occurred that its average values in females were higher than in the control group, however, the difference was not statistically significant $(p=0.57)$. No relation was shown between the uric acid level in psoriatic patients and PASI score, diet, tobacco smoking or family history of dermatosis. In the 
Table 1. Selected parameters of antioxidative and prooxidative factors in the study and reference group

\begin{tabular}{|c|c|c|c|c|c|c|c|c|c|c|c|c|}
\hline \multirow[t]{2}{*}{ Parameter } & \multirow[t]{2}{*}{ Sex } & \multicolumn{5}{|c|}{ Study group } & \multicolumn{5}{|c|}{ Reference group } & \multirow[t]{2}{*}{$P$-value } \\
\hline & & $N$ & Min.-max. & $(\bar{x})$ & SD & $\mathrm{Me}$ & $N$ & Min.-max. & $(\bar{x})$ & SD & $\mathrm{Me}$ & \\
\hline \multirow[t]{3}{*}{ PON-1 [ $\mu \mathrm{mol} / \mathrm{ml}]$} & W & 31 & $17.2-61.4$ & 33.1 & 9.4 & 31.4 & 16 & $40.1-91.3$ & 69.2 & 17.2 & 71.8 & $<0.001$ \\
\hline & M & 35 & $16.2-55.4$ & 33.8 & 11 & 35.4 & 14 & $39.9-90.2$ & 67.4 & 15 & 69.5 & $<0.001$ \\
\hline & All & 66 & $16.2-61.4$ & 33 & 10.2 & 32.1 & 30 & $39.9-91.3$ & 68.3 & 6 & 70.4 & $<0.001$ \\
\hline \multirow{3}{*}{$\begin{array}{l}\alpha \text {-Tocopherol } \\
{[\mathrm{ng} / \mathrm{ml}]}\end{array}$} & W & 31 & $13.5-44.8$ & 25.7 & 9.1 & 23.2 & 16 & $11.1-40.9$ & 27.4 & 8.9 & 29.5 & $>0.05$ \\
\hline & M & 35 & $10.2-40.1$ & 21 & 8.2 & 20.8 & 14 & $12.1-44.2$ & 27.2 & 9.8 & 27.4 & $<0.05$ \\
\hline & All & 66 & $10.2-44.8$ & 23.2 & 8.9 & 21.5 & 30 & $11.1-44.2$ & 27.3 & 9.2 & 28.2 & $<0.05$ \\
\hline \multirow{3}{*}{$\begin{array}{l}\text { Homocysteine } \\
{[\mu \mathrm{mol} / \mathrm{l}]}\end{array}$} & W & 31 & $15.3-46.9$ & 25.4 & 7.9 & 22.4 & 16 & $8.2-20.1$ & 12.8 & 3.4 & 12.3 & $<0.001$ \\
\hline & M & 35 & $19.1-50.1$ & 34.7 & 8.6 & 37.8 & 14 & $9.8-17.9$ & 14.2 & 2.5 & 16.1 & $<0.001$ \\
\hline & All & 66 & $15.3-50.1$ & 30.3 & 9.4 & 29.9 & 30 & $8.2-20.1$ & 13.5 & 3.1 & 13.7 & $<0.001$ \\
\hline \multirow[t]{3}{*}{ Uric acid [mg/dl] } & W & 31 & $3.6-8.1$ & 5.5 & 1.1 & 5.4 & 16 & $3-6.82$ & 4.9 & 1 & 5 & 0.057 \\
\hline & M & 35 & $4.6-11.2$ & 6.7 & 1.3 & 6.3 & 14 & $4.6-7.9$ & 6 & 0.93 & 6.1 & $>0.05$ \\
\hline & All & 66 & $3.6-11.2$ & 6.1 & 1.33 & 6 & 30 & $3.01-7.9$ & 5.4 & 1.13 & 5.4 & $<0.05$ \\
\hline
\end{tabular}

$N$-number of people, $W$-women, $M$ - men, $\bar{x}$ - medium, SD-standard deviation, Me-median, PON-1-paraoxonase-1.

group of patients with psoriasis and metabolic syndrome, the uric acid level was significantly higher than in patients who did not fulfil the syndrome criteria $(p<0.05)$ Moreover, a negative correlation between the uric acid level and abdominal circumference value was revealed $(p<0.001)$. It correlated positively with the levels of homocysteine $(p<0.05)$ and triglycerides $(p<0.05)$.

\section{Discussion}

The presented study showed that PON-1 activity in psoriatic patients was significantly lower as compared to the reference group and it did not correlate with PASI scores, BMI, abdominal circumference or with any other marked risk factors for atherosclerosis. Moreover, there was no relation observed between PON-I activity and dietary habits, tobacco smoking or metabolic syndrome presence.

There are only few available studies on PON-I in psoriatic patients. Most of them were conducted in Asian research centres. The largest of the studies including a group of 100 Iranian patients and 100 healthy individuals showed a decreased PON-I activity in psoriatic patients which correlated positively with the apolipoprotein $A 1$ (apoA1) level and negatively with apolipoprotein B (apoB) and lipoprotein (a) Ip(a) levels [4]. Similar observations were made by Usta et al. [5]. They proved a decreased PON-I activity in psoriatic patients, which additionally correlated with the increased disease severity. In two other studies conducted on a small group of participants, the researchers also observed a decreased PON-I activity in psoriatic patients [6, 7]. Tokera et al. [6] showed no relation between PON-I and PASI; Ramadan et al. [7] described a negative correlation of PON-I activity with the patients' age; they also observed a higher PON-I activity in females.

The only study conducted in Europe by Ferreti et al. in a group of 23 psoriatic patients and 25 healthy individuals revealed a lower PON-I activity in the patients with psoriasis, which correlated negatively with the $\operatorname{lp}(a)$ level and PASI score [8]. The available literature shows that, so far, no study has been published that would describe the PON-I test in psoriatic patients and including a larger group of people in Europe.

A lower PON-I activity in psoriatic patients may be explained by a chronic inflammation accompanying this dermatosis. In order to verify this hypothesis, researchers observed PON-I activity in patients undergoing antipsoriasis treatment. Bacchetti et al. observed changes in biochemical parameters in 23 patients during administration of etanercept [9]. They concluded that with PASI50, PON-I activity and the total antioxidant capacity - TAC increase, whereas the CRP level, which correlates adversely with PON-l, drops. On the other hand, Pektas et al. did not prove any changes in PON-I activity in 24 Turkish psoriatic patients following 30 phototherapy sessions with use of UVB narrowband lamps [10]. Similar observations were made by Kilic et al. who did not report any increase in PON-l activity after 8 weeks of Methotrexate treatment in 28 Turkish patients [11].

The literature includes reports on numerous polymorphisms in PON-I gene that may affect the activity of this enzyme. One of such polymorphisms is L55M which involves a change of TTG codon into ATG in exon 3 of PON-I 
gene. The effect of leucine-to-methionine substitution at position 55 is a lower activity and lower PON-I activity. On the other hand, it seems that this enzyme variant provides a better protection against oxidation [12]. Asefi et al. analysed the presence of this polymorphism in psoriatic patients and confirmed that it is a factor increasing the risk of developing this type of dermatosis, and its presence is related to higher levels of malondialdehyde, apoB, Ip(a) and apoB/apoAl [13]. We proved in the present study, however, that an increased PON-l level occurs in psoriatic patients with a positive family history as compared to patients who do not report any cases of this disease in the family. Hence, it may be concluded that in the Polish population with a family history of psoriasis, polymorphism L55M rather does not occur in psoriatic patients since it is connected with a lower PON-l level.

$\alpha$-Tocopherol is one of four vitamin E forms characterized by the highest biological activity and it is the most common form present in European food products. Tocopherols are synthesized only by photosynthetic organisms and vegetable oils are the richest source of these substances in the diet [14].

In the presented studies, it was revealed that a significantly lower $\alpha$-tocopherol level is observed in psoriatic patients compared to healthy individuals, which is not influenced by the PASI score, dietary habits or metabolic syndrome. Ramadan et al. [7] also reported a lower vitamin $E$ level in patients suffering from chronic diseases, including psoriasis. In another study, the vitamin E level in psoriatic patients was also lower as compared to healthy individuals and it was also dependent on psoriasis severity: the higher the PASI score, the lower $\alpha$-tocopherol level was observed [15]. The literature also includes studies which prove that psoriasis does not affect the vitamin E level [16, 17]. Keihan et al. [18] conducted a study in which they analysed the impact of vitamin E supplementation on the reactions of selected biochemical parameters. They proved that after 3 months of vitamin $E$ administration, the oxLDL level decreases and the PON-I activity in the blood increases. Merzouk et al. [19] established that vitamin E supplementation also lowers levels of total cholesterol, TAG, LDL and apoB. The authors did not observe any influence of vitamin $E$ on a decreased glucose level in blood.

A higher uric acid level was observed in psoriatic patients as compared to healthy individuals. Studies conducted in the United States that included a large group of 27,751 male patients and 71,059 females also confirmed a relation between psoriasis and an increased level of uric acid [20]. Similar observations were made by other authors [21]. The results of our study also showed that the uric acid level was significantly higher in psoriatic patients with metabolic syndrome as compared to the patients who did not fulfil the syndrome criteria. Moreover, the uric acid level correlated positively with an average abdominal circumference and BMI. Kwon et al. believe that a higher uric acid level may result from an increased epithelial cell replacement in patients with psoriatic lesions. This theory is supported by the observation that the higher the PASI score, the higher the uric acid level [22]. Nevertheless, in the present study we established that the uric acid level was independent from PASI score. Our results are contradictory to those obtained in the studies conducted in Asia [23, 24]. The relation between the uric acid level in psoriatic patients depending on their place of residence was described in a meta-analysis by Lai et al. who noticed that in Western Europe there exists a positive correlation between the uric acid level and psoriasis, whereas no similar relation was observed in Eastern Asia and India [24]. On the one hand, these differences may be explained by other race-dependent genetic factors; on the other hand, a different lifestyle and dietary habits of people living on different continents may be the key. Since a higher uric acid level occurs in metabolic syndrome patients and in the group of patients with a higher BMI and a higher average abdominal circumference, the increase does not result from the skin disease only.

The homocysteine level in the present study was significantly higher in the psoriatic patients than in the control group. Its level was not influenced by PASI score, BMI, abdominal circumference, metabolic syndrome, tobacco smoking or lifestyle. Other studies indicate that the homocysteine level, like the uric acid level, depends on the world region where a psoriatic patient lives. Asian studies did not show any substantive differences between the homocysteine level in the study group as compared to the control group [24-26]. In European studies, on the other hand, a significant difference in the homocysteine level is observed between the groups of psoriatic patients and healthy individuals [27-30].

A cause of high homocysteine levels in psoriatic patients may by a genetic predisposition to a higher homocysteine level, e.g. due to inhibited metabolism of this amino acid present in this group of patients. Izmirli et al. [31] proved that in the case of Turkish population, psoriatic patients show a higher frequency of the occurrence of polymorphism in the methylenetetrahydrofolate reductase which takes part in homocysteine metabolism. On the other hand, it is known that in the case of Turkish population, the homocysteine level is not usually increased in psoriasis. The conducted studies also prove that the homocysteine level is lower in the group of patients with a positive family history of psoriasis as compared to patients who do not report any dermatosis cases in their families.

\section{Conclusions}

Psoriasis promotes arteriosclerosis development by decreasing the levels of antiatherogenic and increasing the levels of proatherogenic agents. Adverse changes 
in psoriatic patients involve levels of paraoxonase-1, $\alpha$-tocopherol, uric acid, homocysteine as compared to healthy individuals selected by their age, BMI and abdominal circumference value. The unfavourable profile of these factors is related to the disease itself and the accompanying chronic inflammation. In psoriatic patients, the factors under the study are independent of the patients' age, BMI, tobacco smoking or dietary habits. The uric acid level, on the other hand, correlates positively with the abdominal circumference.

\section{Acknowledgments}

The study was funded by the Medical University of Lodz, project no. 502-03/5-064-01/502-54-171.

\section{Conflict of interest}

The authors declare no conflict of interest.

\section{References}

1. Guo Z, Van Remmen H, Yang H, et al. Changes in expression of antioxidant enzymes affect cell-mediated LDL oxidation and oxidized LDL-induced apoptosis in mouse aortic cells. Arterioscler Thromb Vasc Biol 2001; 21: 1131-8.

2. Tjoelker LW, Wilder C, Eberhardt C, et al. Anti-inflammatory properties of a platelet-activating factor acetylhydrolase. $\mathrm{Na}$ ture 1995; 374: 549-53.

3. Durrington PN, Mackness B, Mackness MI. Paraoxonase and atherosclerosis. Arterioscler Thromb Vasc Biol 2001; 21: 473-80.

4. Houshang N, Reza K, Masoud S, et al. Antioxidant status in patients with psoriasis. Cell Biochem Funct 2014; 32: 268-73.

5. Usta M, Turan E, Aral H, et al. Serum paraoxonase-1 activities and oxidative status in patients with plaque-type psoriasis with/without metabolic syndrome. J Clin Lab Analysis 2011; 25: 289-95.

6. Toker A, Kadi M, Yildirim K, et al. Serum lipid profile paraoxonase and arylesterase activities in psoriasis. Cell Biochem Funct 2009; 27: 176-80.

7. Ramadan S, Tawdy A, Abdel Hay R, et al. The antioxidant role of paraoksonase 1 and vitamin $\mathrm{E}$ in tree autoimmune diseases. Skin Pharmacol Physiol 2013; 26: 2-7.

8. Ferretti G, Bacchetti T, Campanati A, et al. Correlation between lipoprotein(a) and lipid peroxidation in psoriasis: role of the enzyme paraoxonase-1. Br J Dermatol 2012; 166: 204-7.

9. Bacchetti T, Campanati A, Ferreti G, et al. Oxidative stress and psoriasis: the effect of antitumour necrosis factor-alpha inhibitor treatment. Br J Dermatol 2013; 168: 984-9.

10. Pektas SD, Akoglu G, Metin A, et al. Evaluation of systemic oxidant/antioxidant status and paraoxonase 1 enzyme activities in psoriatic patients treated by narrow band ultraviolet B phototherapy. Red Rep 2013; 18: 200-4.

11. Kilic S, Emre S, Metin A, et al. Effect of the systemic use of methotrexate on the oxidative stress and paraoksonase enzyme in psoriasis patients. Arch Dermatol Res 2013; 305: 495500.

12. Mackness M, Mackness B. Human paraoxonase-1 (PON1): gene structure and expression, promiscuous activities and multiple physiological roles. Gene 2015; 567: 12-21.
13. Asefi M, Vaisi-Raygani A, Bahrehmand F, et al. Paraoxonase 1 (PON1) 55 polymorphism, lipid profiles and psoriasis. Br J Dermatol 2012; 167: 1279-86.

14. Duncan KR, Suzuki YJ. Vitamin E nicotinate. Antioxidants 2017; 6: 20.

15. Pujari VM, Ireddy S, Itagi I, Kumar S. The serum levels of malondialdehyde, vitamin $\mathrm{E}$ and erythrocyte catalase activity In psoriasis patients. J Clin Diag Res 2014; 8: 14-6.

16. Shreekantha, Manjunatha BK, Avinash SS, et al. Antioxidant vitamins, calcium and phosphorous levels in psoriasis. Int J Ph B Sci 2010; 1: 208-11.

17. Kokcam I, Naziroglu M. Antioxidants and lipid peroxidation status in the blood of patients with psoriasis. Clin Chim Acta 1999; 289: 23-31.

18. Keihan GS, Gharib MH, Momeni A, et al. A comparison between the effect of cuminum cyminum and vitamin $E$ on the level of leptin, paraoxonase $1, \mathrm{Hbalc}$ and oxidized LDL in diabetic patients. Int J Mol Cell Med 2016; 5: 229-35.

19. Merzouk S, Hichami A, Madani S, et al. Antioxidant status and levels of different vitamins determined by high performance liquid chromatography in diabetic subjects with multiple complications. Gen Physiol Biophys 2003; 22: 15-27.

20. Li X, Miao X, Wang H, et al. Association of serum uric acid levels In psoriasis. A systematic review and meta-analysis. Medicine 2016; 95: e3676.

21. Gisondi P, Targher G, Cagalli A, Girolomoni G. Hyperuricemia in patients with chronic plaque psoriasis. I Am Acad Derm 2014; 70: 127-30.

22. Kwon HH, Kwon IH, Choi JW, Youn JI. Cross-sectional study on the correlation of serum uric acid with disease severity In Korean patients with psoriasis. Clin Exp Dermatol 2011; 36: 473-38.

23. Merola JF, Wu S, Han J, et al. Psoriasis, psoriatic arthritis and risk of gout In US men and women. Ann Rheum Dis 2015; 74: 1495-500.

24. Lai YC, Yew YW. Psoriasis and uric acid: a population-based cross-sectional study. Clin Exp Derm 2016; 41: 260-6.

25. Ataseven A, Kesli R, Kurtipek GS, Oztuk P. Assessment of lipocalin 2, clusterin, soluble tumor necrosis factor receptor-1, interleukin-6, homocysteine, and uric acid levels In patients with psoriasis. Disease Markers 2014; 2014: e541709.

26. Erturan I, Koroglu BK, Adiloglu A, et al. Evaluation of serum SCD40L and homocysteine levels with subclinical atherosclerosis indicators In patients with psoriasis: a pilot study. Int J Derm 2014; 53: 503-9.

27. Tobin AM, Hughes R, Hand EB, et al. Homocysteine status and cardiovascular risk factors in patients with psoriasis: a casecontrol study. Clin Exp Derm 2010; 36: 19-23.

28. Gianotti M, Consales V, Campanati A, et al. Homocysteine plasma levels in psoriasis patients: our experience and review of the literature. J Eur Acad Derm Venereol 2015; 29: 1781-85.

29. Vanizor KB, Orem A, Cimsit, et al. Plasma homocysteine and its relationships with atherothrombotic markers in psoriatic patients. Clin Chim Acta 2003; 332: 23-30.

30. Armitage JM, Bowman L, Clarke RJ, et al. Effects of homocysteine-lowering with folic acid plus vitamin B12 vs. placebo on mortality and major morbidity in myocardial infarction survivors: a randomized trial. J Am Med Associat 2010; 303: 2486-94.

31. Izmirli M, Bulbul Sen B, Rifaioglu E, et al. Methylenetetrahydrofolate reductase (MTHFR) C677T polymorphism in psoriasis in southern Turkey. An Bras Dermatol 2016; 91: 611-3. 